\title{
Clinical Outcome of Reconstruction Using Frozen Autograft for a Humeral Bone Tumor
}

\author{
HIROAKI KIMURA, NORIO YAMAMOTO, TOSHIHARU SHIRAI, HIDEJI NISHIDA, KATSUHIRO HAYASHI, \\ YOSHIKAZU TANZAWA, AKIHIKO TAKEUCHI, SHINJI MIWA and HIROYUKI TSUCHIYA
}

\author{
Department of Orthopedic Surgery, Kanazawa University, Kanazawa, Japan
}

\begin{abstract}
Background: We developed a frozen autograft technique in which a tumor-bearing bone was treated with liquid nitrogen for reconstruction. The purpose of this study was to evaluate the functional outcomes of this technique after resection of humeral bone tumors. Patients and Methods: Eight patients were included in the study. The freezing range was classified into 2 groups, depending on whether the joint surface of the humeral head was frozen. Results: In the joint freezing group, the mean active shoulder abduction and flexion were $43.8^{\circ}$ and $55.0^{\circ}$, and the mean International Society of Limb Salvage (ISOLS) score was $70.8 \%$. In the joint preservation group, the mean active shoulder abduction and flexion were $130^{\circ}$ and $132^{\circ}$, and the mean ISOLS score was 90.8\%. Progressive and massive bone absorption was seen in 3 cases. Discussion: Reconstruction using frozen autograft after resection of humeral bone tumor is our preferred option, especially in shoulder joint preservation surgery.
\end{abstract}

Reconstruction of humeral bone loss resulting from tumor resection remains challenging, although limb-saving surgeries have become preferred treatment options for their cosmetic and functional advantages. Even though endoprosthetic reconstruction is commonly used, the functional outcome is often unsatisfactory (1). Biological reconstruction is favored over endoprosthetic reconstruction in terms of functional results because gleno-humeral instability is considered less frequent in patients with biological reconstruction $(2,3)$.

The method of recycling bone consists of various treatments, such as autoclaving (4), irradiation (5), and pasteurization (6). Since this method uses the patient's resected bone, the bone's size and shape can be adaptable,

Correspondence to: Hiroyuki Tsuchiya, 13-1 Takaramachi, Kanazawa, Ishikawa 920-8641, Japan. Tel: +81 762652374, Fax: +81 762344261, e-mail: tsuchi@med.kanazawa-u.ac.jp.

Key Words: Humeral bone tumor, biological reconstruction, frozen autograft. and there is no risk of disease transmission nor immunologic reaction. As with allograft, these techniques cause thermalor radiation-induced weakness of bone with loss of osteoinductive properties (7), resulting in irreparable fracture, severe bone absorption, and early revision surgery. Combined with vascularized fibula graft, it reportedly reduces the complication rate of recycled bone graft, although it requires a long operation time $(8,9)$.

We developed a frozen autograft technique, and we have utilized this technique to perform reconstruction after malignant bone tumor resection since 1999. Liquid nitrogen at $-196^{\circ} \mathrm{C}$ is used as a cryogenic agent to destroy tumor cells, and freezing devitalizes tumor cells by inducing ice crystal formation and cell dehydration. This method was developed based on in vitro and in vivo studies of the hypothermic effects of liquid nitrogen on the proliferation of osteosarcoma cells (10). The advantages of using frozen autografts are as follows: they are simple, they require a short treatment time, they make a perfect fit, they have sufficient biochemical strength, there is no need for bone banking, they are easy to attach to tendons and ligaments, they have cryo-immunological activity, and both osteoinductive and osteoconductive properties are preserved. Takata et al. reported that the activity of bone morphogenetic proteins was better preserved in liquid nitrogen treatment compared to autoclaving or pasteurization (11), and that revitalization of grafted frozen bone was observed as well. Bone revitalization after freezing was observed at a relatively early stage following transplantation (12). Therefore, we think that frozen autografting does not require for additional procedures, such as vascularized fibula grafting.

For humeral reconstruction following malignancy, we also used frozen autografts to achieve good function and preserve bone stock. The present study aimed to evaluate functional outcomes and complications in patients treated with frozen autograft after the resection of humeral malignant or aggressive benign bone tumors, and describe the benefits and problems associated with this method and our current technique. 


\section{Free freezing}
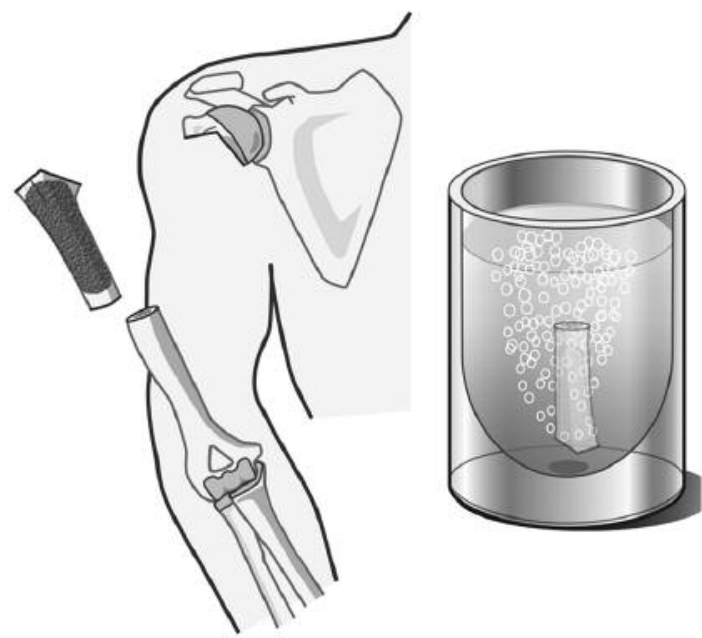

Figure 1. Schematic diagram of free and pedicle freezing procedures.

\section{Patients and Methods}

Patients. Between 1999 and 2012, 12 patients with malignant or aggressive benign tumors of the humerus underwent reconstruction surgery with frozen autograft. We excluded bone tumors with severe osteolytic change, because frozen autograft with a huge cortical defect does not have sufficient strength for reconstruction.

Of 12 patients, eight with a minimum two-year follow-up postoperatively were evaluated (three men and five women; average age at diagnosis, 38.5 years; range $=12-78$ years). Regarding their histological diagnosis, five of these cases were osteosarcomas, one chondrosarcoma, one multiple myeloma, and one phosphaturic mesenchymal tumor (an FGF23-producing tumor). Four patients with primary high-grade osteosarcoma received neoadjuvant chemotherapy based on the K2 protocol (13), and 2-3 weeks postoperatively, chemotherapy was resumed.

Resection and freezing procedures. Resection of the tumor was performed with a wide margin or intentional marginal margin (14), including the biopsy tract and a sufficient cuff of normal tissue all around the tumor. V-shape osteotomy of the bone was performed to increase stability and the surface contact area. The extraosseous tissue was removed, leaving important muscle insertions, ligaments, and joint capsule for reattachment. Then the intramedullary canals were curetted to remove the bone marrow and tumor content to prevent graft fracture due to water volume expansion during freezing.

The freezing procedure has previously been described (15). The frozen autograft was replaced to its original position with reconstruction. Cancellous bone graft or bone cement was used for mechanical support, if necessary. If the tendons or ligaments were left on the bone, they were sutured to the original corresponding structures.

The developers of this technique also modified it to produce a pedicle frozen autograft to maintain anatomical continuity on one

\section{Pedicle freezing}

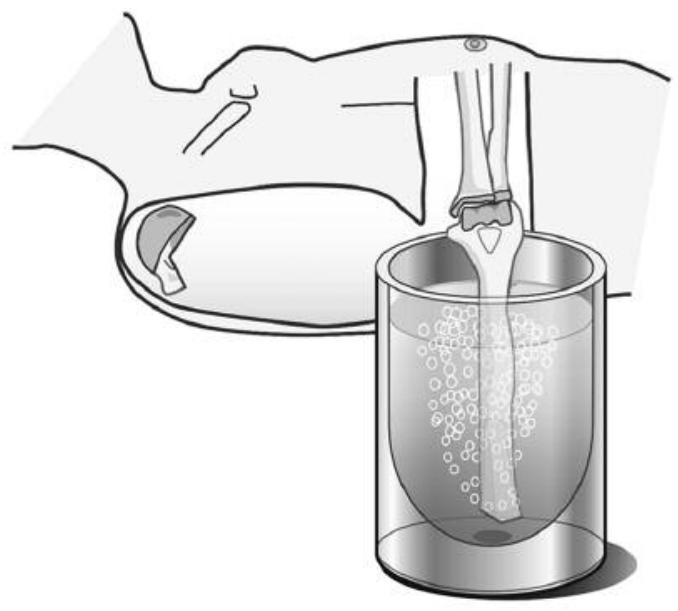

side (Figure 1) (16). Specifically, V-shape osteotomy was performed $2 \mathrm{~cm}$ from the proximal side of the bony margin. The soft tissue was divided along the extremity beyond the surface of the surgical margin until the arc of rotation was adequate. Surgical sheets protected the surrounding soft tissue. Bony lesions connected to the limb were carefully rotated and frozen in liquid nitrogen while the surrounding soft tissue was still protected with surgical sheets. The part of the bone $2 \mathrm{~cm}$ away from the bony margin was positioned at the surface level of the liquid nitrogen. Using this method, cutting both sides of the tumor site is unnecessary; thus, it provides the following advantages: the preservation of joint stability and function in selected patients, because no important ligaments need to be sacrificed; fewer osteotomy sites; the preservation of initial strength at the host-graft junction; and a lower rate of graft healing complications because of early blood flow recovery (17). We performed free freezing methods in the first two cases, while pedicle freezing methods were used in the other six cases. After the frozen autograft was replaced, resected muscle insertions, ligaments, and the joint capsule were reattached to the original corresponding structures as best as possible.

According to the International Society of Limb Salvage (ISOLS) resection classification system (Figure 2) (18), the freezing ranges of the humerus were classified as S34 in two patients, S345 in two patients, and S45 in four patients. No patient who underwent scapulectomy (S1 or $\mathrm{S} 2$ resection) was included. This may be because we excluded cases with severe osteolytic change, in which significant extraosseous invasion or intra-articular invasion of the tumor often occurred. For functional evaluation, the freezing range was classified into two groups, based on whether the joint surface of the humeral head (S3 region) was frozen or not: the joint freezing group and the joint preservation group with four patients each. We evaluated the reconstruction methods, range of motion (ROM) of the shoulder joint, function of the upper extremities, bone union at the osteotomy site, bone absorption of the frozen bone, and complications of each group. Function at the final follow-up assessment was evaluated using the 


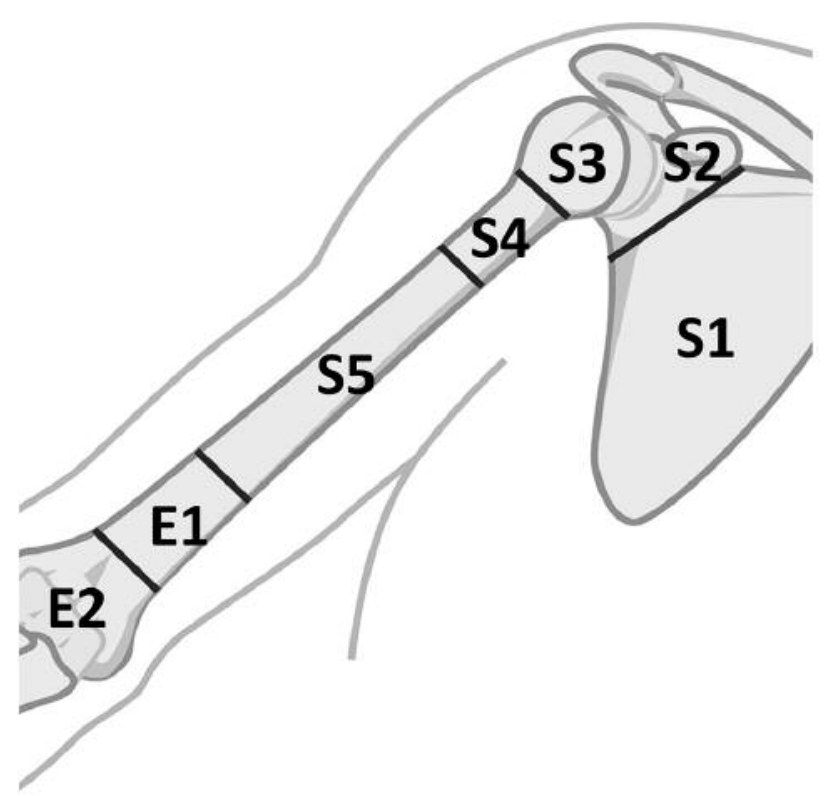

Figure 2. Classification of the ISOLS for shoulder girdle resection.

ISOLS/Musculoskeletal Tumor Society (MSTS) scoring system (19). Bone union was defined as the presence of indistinct bone incision lines or bridging callus on radiographs.

This study was approved by the institutional review board. All patients and/or their family gave consent to participate.

\section{Results}

For eight patients, the mean postoperative follow-up period was 78.5 months (range $=25-181$ months). The oncological outcomes were continuously disease-free in four cases, no evidence of disease in two cases, alive with disease in one case, and died of disease in one case. No local recurrence was observed (Table I).

In the joint freezing group, no case underwent extraarticular resection. All cases underwent intra-articular resection and mutilation of rotator cuff and joint capsule. The first two cases were reconstructed with osteoarticular graft, and fixation was performed with intramedullary nailing and bone cement; osteochondral necrosis with collapse of the subchondral bone and sequential severe bone absorption in the humeral head occurred in both of these cases. So the latter two cases were reconstructed with a frozen autograft prosthesis composite. The mean active shoulder abduction and flexion was $43.8^{\circ}$ and $55.0^{\circ}$, respectively. The mean ISOLS/MSTS score was $70.8 \%$.

In the joint preservation group, fixation with intramedullary nailing and bone cement was performed in 2 cases, and fixation with plating was used in the latter 2 cases. The mean active shoulder abduction and flexion were $130^{\circ}$

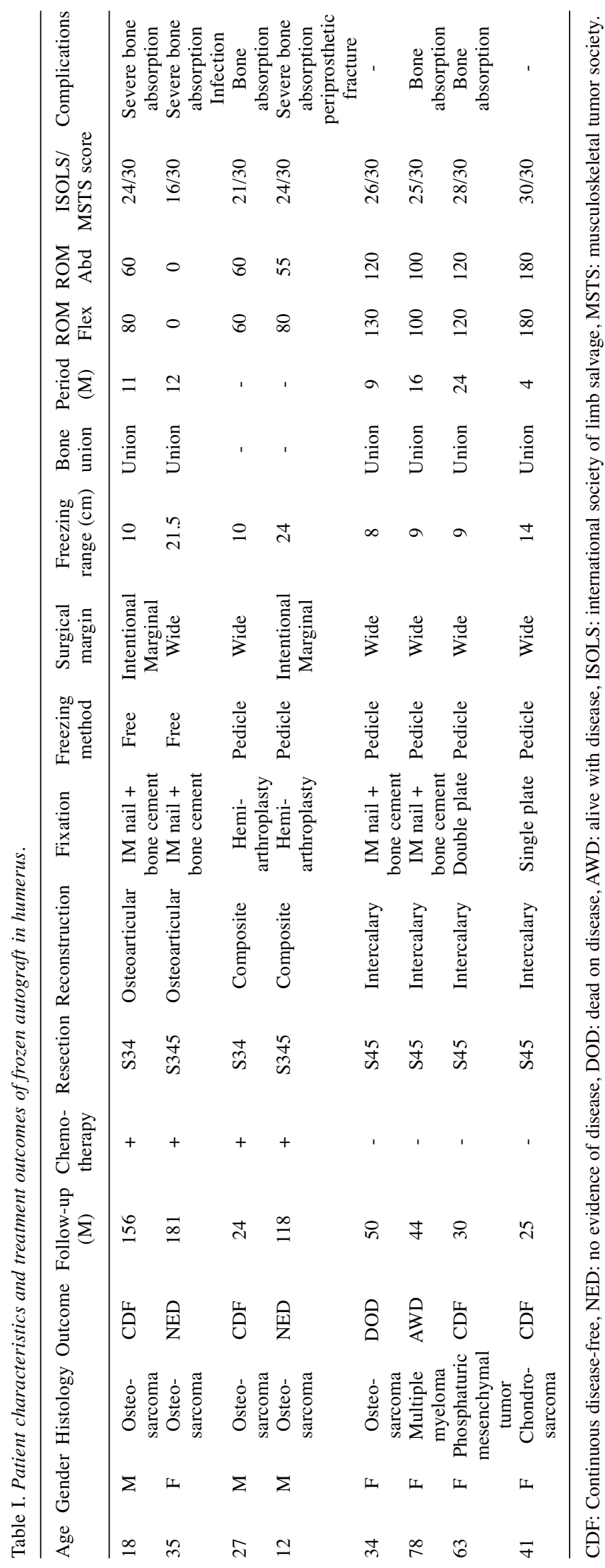


Table II. Functional outcome of joint freezing group and joint preservation group.

\begin{tabular}{|c|c|c|c|c|}
\hline & Reconstruction & ROM abduction & ROM flexion & ISOLS/MSTS score \\
\hline Joint freezing & Osteoarticular & $43.8^{\circ}$ & $55.0^{\circ}$ & $70.8 \%$ \\
\hline \multirow[t]{3}{*}{$\mathrm{N}=4$} & $\mathrm{~N}=2$ & $\left(0^{\circ}-60^{\circ}\right)$ & $\left(0^{\circ}-80^{\circ}\right)$ & $(53.3-80.0)$ \\
\hline & Composite & & & \\
\hline & $\mathrm{N}=2$ & & & \\
\hline Joint preservation & IM nail with bone cement & $130^{\circ}$ & $132^{\circ}$ & $90.8 \%$ \\
\hline \multirow[t]{3}{*}{$\mathrm{N}=4$} & $\mathrm{~N}=2$ & $\left(100^{\circ}-180^{\circ}\right)$ & $\left(100^{\circ}-180^{\circ}\right)$ & $(83.3-100)$ \\
\hline & Double plate & & & \\
\hline & $\mathrm{N}=2$ & & & \\
\hline
\end{tabular}

and $132^{\circ}$, respectively. The mean ISOLS/MSTS score was $90.8 \%$, representing a good functional outcome (Table II).

Six patients who had at least one osteotomy site were available for the evaluation of bone union. Bone union at the final follow-up was achieved in all cases. The mean union period was 12.7 months (range $=4-24$ months). This period was relatively long compared to all the frozen autograft data of the upper and lower extremities, and pelvis (20).

Bone absorption of frozen autograft was observed in six of eight cases. Progressive and massive bone absorption was observed in three cases that had osteoarticular frozen autograft, underwent the free freezing procedure, or had a bone freezing range over $20 \mathrm{~cm}$. In the other three cases, there was slight bone absorption that stopped progressing within 12 months, and it did not go on to develop symptomatic problems. In one case with massive bone absorption, additional surgery was required because of protrusion of the intramedullary nail. The remaining two patients with massive bone absorption were managed conservatively.

Complications, except for bone absorption, were encountered in two of eight patients. Deep infection occurred in one case, that was successfully treated with irrigation, debridement, and a latissimus dorsi flap. Periprosthetic fracture at the distal end of the prosthesis occurred in one case, which was cured conservatively. There was only one failure due to progressive bone absorption that necessitated removal of the construct. The survival rate of frozen autograft was $87.5 \%$ (seven patients).

\section{Discussion}

Limb function following tumor resection around the humerus is often limited by surgical loss of surrounding functional soft tissue stabilizers of the gleno-humeral joint. Although the deltoid muscle and axillary nerve can be spared without compromising oncological surgical margins, resection of a transarticular tumor, including part of the deltoid muscle and rotator cuff, are usually inevitable, resulting in instability of the gleno-humeral joint and muscle weakness of shoulder motion.
To achieve good stability and function after transarticular proximal humeral resection, the destroyed rotator cuff and muscle should be reconstructed even if they will not be functional again. Therefore, biological reconstruction has an advantage compared to endoprosthetic reconstruction.

Biological reconstruction is the best surgical approach, because it preserves gleno-humeral stability, is associated with a comparable complication rate, and has a long durability. The limitation to bone recycling reconstruction, including frozen bone graft, is that the tumor-bearing bone with strong osteolytic change cannot be used for reconstruction without any mechanical reinforcement. Thus, the best candidates for bone recycling reconstruction are patients with osteosarcoma who have received preoperative chemotherapy, achieved a good response, and have osteosclerotic change in the tumor lesion.

In our study, the freezing method resulted in loss of ROM and unsatisfactory upper limb function. Loss of the abductor mechanism is a common issue in all proximal humeral reconstruction procedures. De Wilde et al. reported excellent abduction ROM after reconstruction with reverse shoulder arthroplasty (RSA)-irradiated bone composite (21). Although rotator cuff function was lost, function with abduction and forward flexion of $>140^{\circ}$ can be expected after reconstruction with RSA. However, they also reported severe resorption of grafted irradiated bone. An RSA-frozen graft composite may be the best option for transarticular proximal humeral resection.

Our study also showed that a bone freezing range $>20 \mathrm{~cm}$, the free freezing method, and an osteoarticular graft resulted in progressive bone absorption. Our current technique for performing reconstruction with frozen autograft after resection of the humeral bone tumor is as follows.

1. Perform pedicle freezing as much as possible.

2. If joint freezing is required, use a frozen autograft prosthesis composite instead of an osteoarticular graft.

3. Perform plate fixation without cementation instead of intramedullary nailing with bone cement when joint preservation surgery is available because of the benefit of early revitalization of frozen bone. 
4. Use devices in combination with vascularized fibula graft if the bone freezing range is $>20 \mathrm{~cm}$.

The limitations of this study were its small cohort and the selection bias of patients with less osteolytic change. Less osteolytic change is often associated with less extraosseous tumor invasion. This is directly related to the surgical margin and postoperative functional results, and it is one of the reasons why our series did not include patients who required resection of the neurovascular bundle or extra-articular resection.

In conclusion, reconstruction with frozen autograft following tumor resection of malignant or aggressive benign tumors of the humerus is our preferred option because of its acceptable complication rate. The functional outcome is strongly influenced by whether the joint surface of the humeral head is treated. Bone absorption of frozen bone was associated with a bone freezing range $>20 \mathrm{~cm}$, the free freezing method, and osteoarticular graft. We recommend our current technique for performing reconstruction of a humeral bone tumor with frozen autograft.

\section{References}

1 Cannon CP, Paraliticci GU, Lin PP, Lewis VO and Yasko AW: Functional outcome following endoprosthetic reconstruction of the proximal humerus. J Shoulder Elb Surg 18: 705-710, 2009.

2 Abdeen A and Healey JH: Allograft-prosthesis composite reconstruction of the proximal part of the humerus: surgical technique. J Bone Joint Surg Am 92: 188-196, 2010.

3 Potter BK, Adams SC, Pitcher JD, Malinin TI and Temple HT: Proximal humerus reconstructions for tumors. Clin Orthop Relat Res 467: 1035-1041, 2009.

4 Asada N, Tsuchiya H, Kitaoka K, Mori Y and Tomita K: Massive autoclaved allografts and autografts for limb salvage surgery. A 1-8 year follow-up of 23 patients. Acta Orthop Scand 68: 392-395, 1997.

5 Araki N, Myoui A, Kuratsu S, Hashimoto N, Inoue T, Kudawara I, Ueda T, Yoshikawa H, Masaki N and Uchida A: Intraoperative extracorporeal autogenous irradiated bone grafts in tumor surgery. Clin Orthop Relat Res 368: 196-206, 1999.

6 Manabe J, Ahmed AR, Kawaguchi N, Matsumoto S and Kuroda $\mathrm{H}$ : Pasteurized autologous bone graft in surgery for bone and soft tissue sarcoma. Clin Orthop Relat Res 419: 258-266, 2004.

7 Singh VA, Nagalingam J, Saad M and Pailoor J: Which is the best method of sterilization of tumour bone for reimplantation? A biomechanical and histopathological study. Biomed Eng Online 9: 48, 2010

8 Sugiura H, Takahashi M, Nakanishi K, Nishida Y and Kamei Y: Pasteurized intercalary autogenous bone graft combined with vascularized fibula. Clin Orthop Relat Res 456: 196-202, 2007.

9 Muramatsu K, Ihara K, Miyoshi T, Yoshida K, Iwanaga R, Hashimoto T and Taguchi T: Stimulation of neo-angiogenesis by combined use of irradiated and vascularized living bone graft for oncological reconstruction. Surgical Oncol 21: 223-229, 2012.
10 Yamamoto N, Tsuchiya $\mathrm{H}$ and Tomita K: Effects of liquid nitrogen treatment on the proliferation of osteosarcoma and the biomechanical properties of normal bone. J Orthop Sci 8: 374380, 2003.

11 Takata M, Sugimoto N, Yamamoto N, Shirai T, Hayashi K, Nishida H, Tanzawa Y, Kimura H, Miwa S, Takeuchi A and Tsuchiya $\mathrm{H}$ : Activity of bone morphogenetic protein-7 after treatment at various temperatures: Freezing $v s$. pasteurization $v s$. allograft. Cryobiology 63: 235-239, 2011.

12 Tanzawa Y, Tsuchiya H, Shirai T, Hayashi K, Yo Z and Tomita $\mathrm{K}$ : Histological examination of frozen autograft treated by liquid nitrogen removed after implantation. J Orthop Sci 14: 761-768, 2009.

13 Tsuchiya H, Tomita K, Yamamoto N, Mori Y and Asada N: Caffeine-potentiated chemotherapy and conservative surgery for high-grade soft-tissue sarcoma. Anticancer Res 18: 3651-3656, 1998.

14 Tsuchiya H, Tomita K, Mori Y, Asada N and Yamamoto N: Marginal excision for osteosarcoma with caffeine assisted chemotherapy. Clin Orthop Relat Res 358: 27-35, 1999.

15 Tsuchiya H, Wan SL, Sakayama K, Yamamoto N, Nishida H and Tomita K: Reconstruction using an autograft containing tumour treated by liquid nitrogen. J Bone Joint Surg Br 87: 218-225, 2005.

16 Tsuchiya H, Nishida H, Srisawat P, Shirai T, Hayashi K, Takeuchi A, Yamamoto $\mathrm{N}$ and Tomita $\mathrm{K}$ : Pedicle frozen autograft reconstruction in malignant bone tumors. J Orthop Sci 15: 340-349, 2010.

17 Shimozaki S, Yamamoto N, Shirai T, Nishida H, Hayashi K, Tanzawa Y, Kimura H, Takeuchi A, Igarashi K, Inatani H, Kato $\mathrm{T}$ and Tsuchiya $\mathrm{H}$ : Pedicle versus free frozen autograft for reconstruction in malignant bone and soft tissue tumors of the lower extremities. J Orthop Sci 19: 156-163, 2014.

18 Enneking W, Dunham W, Gebhardt M, Malawar M and Pritchard D: A system for the classification of skeletal resections. Chir Organi Mov 75: 217-240, 1990.

19 Enneking WF, Dunham W, Gebhardt MC, Malawar M and Pritchard DJ: A System for the Functional-Evaluation of Reconstructive Procedures after Surgical-Treatment of Tumors of the Musculoskeletal System. Clin Orthop Relat Res 286: 241246, 1993.

20 Igarashi K, Yamamoto N, Shirai T, Hayashi K, Nishida H, Kimura H, Takeuchi A and Tsuchiya H: The long-term outcome following the use of frozen autograft treated with liquid nitrogen in the management of bone and soft-tissue sarcomas. Bone Jt J 96: 555-561, 2014.

21 De Wilde L, Boileau P and Van Der Bracht H: Does reverse shoulder arthroplasty for tumors of the proximal humerus reduce impairment? Clin Orthop Relat Res 469: 2489-2495, 2011.

Received October 9, 2016

Revised October 26, 2016

Accepted November 1, 2016 